\title{
Wetting of liquid-crystal surfaces and induced smectic layering at a nematic-liquid interface: An x-ray reflectivity study
}

\author{
Masafumi Fukuto, ${ }^{1, *}$ Oleg Gang, ${ }^{2}$ Kyle J. Alvine, ${ }^{3}$ Benjamin M. Ocko, ${ }^{1}$ and Peter S. Pershan,${ }^{3,4}$ \\ ${ }^{1}$ Condensed Matter Physics and Materials Science Department, Brookhaven National Laboratory, Upton, New York 11973, USA \\ ${ }^{2}$ Center for Functional Nanomaterials, Brookhaven National Laboratory, Upton, New York 11973, USA \\ ${ }^{3}$ Division of Engineering and Applied Sciences, Harvard University, Cambridge, Massachusetts 02138, USA \\ ${ }^{4}$ Department of Physics, Harvard University, Cambridge, Massachusetts 02138, USA
}

(Received 17 December 2007; revised manuscript received 6 February 2008; published 14 March 2008)

\begin{abstract}
We report the results of a synchrotron x-ray reflectivity study of bulk liquid-crystal surfaces that are coated by thin wetting films of an immiscible liquid. The liquid-crystal subphase consisted of the nematic or isotropic phase of 4-octyl-4' -cyanobiphenyl (8CB), and the wetting film was formed by the fluorocarbon perfluoromethylcyclohexane (PFMC), a volatile liquid. The thickness of the wetting film was controlled by the temperature difference $\Delta T_{\mu}$ between the sample and a reservoir of bulk PFMC, contained within the sealed sample cell. Phase information on the interfacial electron density profiles has been extracted from the interference between the scattering from the PFMC-vapor interface and the surface-induced smectic order of the $8 \mathrm{CB}$ subphase. The liquid-crystal side of the nematic-liquid (8CB-PFMC) interface is characterized by a density oscillation whose period corresponds to the smectic layer spacing and whose amplitude decays exponentially toward the underlying nematic subphase. The decay length $\xi$ of the smectic amplitude is independent of the PFMC film thickness but increases as the nematic-smectic- $A$ transition temperature $T_{\mathrm{NA}}$ is approached, in agreement with the longitudinal correlation length $\xi_{\|} \propto\left(T-T_{\mathrm{NA}}\right)^{-0.7}$ for the smectic fluctuations in the bulk nematic. The results indicate that the homeotropic orientation of the $8 \mathrm{CB}$ molecules is preferred at the $8 \mathrm{CB}$-PFMC interface and that the observed temperature dependence of the smectic layer growth is consistent with the critical adsorption mechanism. The observed $\Delta T_{\mu}$ dependence of the PFMC film thickness, $L \propto\left(\Delta T_{\mu}\right)^{-1 / 3}$, implies that PFMC completely wets the $8 \mathrm{CB}$ surface and is dominated by the nonretarded dispersion interactions between hydroand fluorocarbons. The complete wetting behavior of PFMC is nearly independent of the degree of interfacial smectic order in the subphase.
\end{abstract}

DOI: 10.1103/PhysRevE.77.031607

PACS number(s): 68.15.+e, 61.30.Hn, 61.05.cm

\section{INTRODUCTION}

Fluid interfaces and films often provide an ideal platform for exploring fundamental aspects of condensed matter. The fluid interfacial phenomena that have been studied extensively for this purpose include wetting [1-4], surface freezing [5], and surface layering [6-13]. Surface-induced ordering provides a means to control the molecular orientation in liquid crystals. For example, there is a growing interest in using fluorinated surfaces to align columnar phases of discotic liquid crystals $[14,15]$. This is motivated, e.g., by the importance of homeotropic (normal-to-surface) alignment of the columnar director to the development of efficient organic photovoltaic devices [14-16]. In order to facilitate the rational design of alignment surfaces, it is essential to improve our fundamental understanding of how a given interfacial material causes liquid-crystal molecules to adopt a particular orientation. Due to its simplicity and relative lack of defects, the interface between a liquid crystal and an ordinary liquid should be well suited for elucidating the effects of contact material on liquid crystals [17].

Composite systems involving both liquids and liquid crystals have recently been drawing a great deal of attention. Dispersions of micrometer-scale liquid droplets within nematic films $[18,19]$ or at nematic-air interfaces [20] have been

\footnotetext{
*fukuto@bnl.gov
}

used as model systems for studying the interparticle interactions that are mediated by nematic director fields. Inverse systems, i.e., suspensions of nematic droplets in liquids have been studied for their potential as the working component in optoelectronic devices like displays and light shutters [21]. With a goal of developing label-free biosensors, the sensitivity of liquid crystals to boundary conditions has been exploited to optically detect the adsorption of lipids and proteins at nematic-aqueous-buffer interfaces [22]. While liquid-liquid crystal interfaces are an essential feature of all these systems, direct structural measurements of these interfaces have been rather limited [17].

We present here a synchrotron $\mathrm{x}$-ray reflectivity (XR) study of bulk liquid crystal surfaces that are coated by thin wetting films of a fluorocarbon liquid. We chose 4-octyl$4^{\prime}$-cyanobiphenyl $(8 \mathrm{CB})$ as the liquid-crystalline subphase because smectic layering had already been observed at both vapor and solid (but not liquid) interfaces of the bulk nematic or isotropic phases of $8 \mathrm{CB}$ and related analogs [6-13]. The wetting film was formed by the fluorocarbon perfluoromethylcyclohexane (PFMC), which has been shown to completely (and immiscibly) wet the surface of a long-chain hydrocarbon liquid [23,24].

Our principal objective is to elucidate the effects of a PFMC overlayer on the interface-induced smectic layering behavior of nematic $8 \mathrm{CB}$. In addition to addressing the role of the fluorocarbon in aligning liquid crystals, the present study allows us to examine the behavior of a liquid crystal at 
an interface with a liquid [17]. In particular, interference between the reflections from the liquid-vapor interface and the underlying liquid crystal provides more definitive phase information on the induced smectic order than was available in the previous measurements on the free surface of other liquid crystals $[7,9,10]$. On the basis of the electron density profiles extracted from XR, we will show that the homeotropic orientation is preferred for the $8 \mathrm{CB}$ molecules that are in contact with the fluorocarbon; this in turn induces critical adsorption of smectic layers at the nematic-8CB-PFMC interface. The results will be contrasted with our previous observation that the adsorption of PFMC completely suppresses the surface freezing of an $n$-alkane [23].

The second objective is to extend the study of wetting phenomena to those involving a liquid-crystalline subphase. The surface of a liquid crystal near a bulk phase transition provides an opportunity to study how the wetting behavior may be influenced by the variable degree of structural order just below the interface. Our results indicate that PFMC completely wets the $8 \mathrm{CB}$ surface and that the thickening of PFMC films with reduced chemical potential is nearly independent of the degree of induced smectic layering on the $8 \mathrm{CB}$ side of the interface.

This paper is organized as follows. Section II provides experimental details. Section III presents the results of XR measurements and data analysis (Sec. III A) and discussions of induced smectic layering (Sec. III B) and wetting behavior (Sec. III C). Section IV summarizes the main conclusions.

\section{EXPERIMENTAL DETAILS}

\section{A. X-ray measurements}

$\mathrm{X}$-ray experiments were carried out at beamline X22B of the National Synchrotron Light Source, using the HarvardBNL liquid surface spectrometer [25] (x-ray wavelength $\lambda$ $=1.53 \AA$ A $)$. The relationship between the horizontal sample surface ( $x-y$ plane) and the scattering angles $(\alpha, \beta, \psi)$ is illustrated in Fig. 1(a). The difference between the scattered and incident wave vectors defines the wave vector transfer $\mathbf{q}$ $=\mathbf{k}_{\text {out }}-\mathbf{k}_{\text {in }}$. Its surface-normal ( $z$ axis) and surface-parallel components are given by $q_{z}=k[\sin (\alpha)+\sin (\beta)], \quad q_{x}$ $=k \cos (\beta) \sin (\psi)$, and $q_{y}=k[\cos (\beta) \cos (\psi)-\cos (\alpha)]$, respectively, where $k=2 \pi / \lambda$. In XR, the intensity $I$ reflected from the surface at the specular condition $\left(\beta=\alpha, \psi=0 ; q_{x y}=0\right)$ is measured as a function of the incident angle $\alpha$ or wave vector transfer $q_{z}=2 k \sin (\alpha)$; see Fig. 1(b). Typical detector slit settings corresponded to angular acceptance of $\Delta \beta=0.14^{\circ}$ and $\Delta \psi=0.14^{\circ}$, or, equivalently, reciprocal-space resolutions of $\quad \Delta q_{x} \approx k \Delta \psi=0.010 \AA^{-1}, \quad \Delta q_{y} \approx k \sin (\beta) \Delta \beta=\left(q_{z} / 2\right) \Delta \beta$ $=0.0013 q_{z}$, and $\Delta q_{z} \approx k \Delta \beta=0.010 \AA^{-1}$. The reported signal is the difference between the intensity measured at the specular position $(\psi=0)$ and the background intensity measured at $\psi$ offsets of $\psi_{b}= \pm 0.15^{\circ}$. The background-subtracted intensity was normalized to the incident beam to obtain the reflectivity $R\left(q_{z}\right)=I\left(q_{z}\right) / I_{0}$. For the surfaces and settings used in the present study, the contribution of the capillary-wave diffuse scattering to the measured background is insignificant as compared to the specular contribution and therefore neglected in the analysis below. (a)
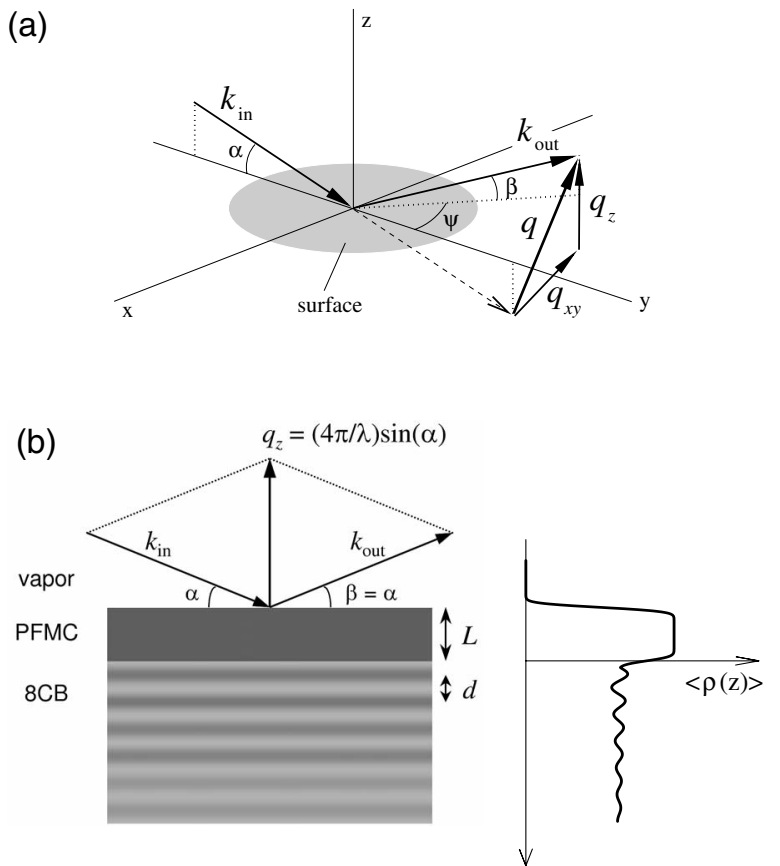

FIG. 1. (a) Surface x-ray scattering geometry. (b) Specular x-ray reflectivity geometry and an illustration of a wetting film (PFMC) of thickness $L$ on the surface of a bulk liquid crystal (8CB) with induced smectic layers of spacing $d$. The average electron density profile $\langle\rho(z)\rangle$ along the interface normal is shown on the right.

\section{B. Wetting chamber, materials, and sample preparation}

The wetting chamber $[23,24]$ consisted of two concentric temperature-controlled cells. The hermetically sealed inner copper cell contained the sample (PFMC film on a puddle of $8 \mathrm{CB})$ in the middle and a bulk reservoir of wetting liquid (PFMC) at the bottom, such that the surface to be wetted was approximately $\sim 50 \mathrm{~mm}$ above the cell bottom. The thickness of the wetting film was varied by controlling the temperature difference $\Delta T_{\mu}=T-T_{\text {res }}>0$ between the sample and the reservoir [23,24,26-29], using the setup described in Ref. [24].

PFMC (Fluka, 97\%) and 8CB (Frinton Laboratories, 99\%) were used as received. The liquid crystal was supported on a flat Pyrex disk (Swift Glass Co., $51 \mathrm{~mm}$ diameter, $1 \mathrm{~mm}$ thick, parallel ground, and polished) that was cleaned and then treated with $N, N$-dimethyl- $N$-octadecyl-3aminopropyltrimethoxysilyl chloride (DMOAP; United Chemical Technologies) [10,11,30].

The inner cell and internal parts were cleaned as described previously [24]. The DMOAP-coated Pyrex flat was mounted onto a copper disk with a shallow circular trough such that the glass fitted closely in the trough and was pressed against the copper surface for good thermal contact. The copper trough had been cleaned with organic solvents and a 3-min soak in a solution of $\mathrm{H}_{2} \mathrm{SO}_{4}, \mathrm{HNO}_{3}$, and water (20\%:10\%:70\% by volume), followed by a thorough rinse with ultrapure water and drying on a heating plate. With the mounted sample holder in place, the sample platform was heated above the nematic-isotropic (NI) transition temperature $T_{\mathrm{NI}} \approx 40{ }^{\circ} \mathrm{C}$ of $8 \mathrm{CB}[13,31-36]$, and roughly $1 \mathrm{~g}$ of $8 \mathrm{CB}$ 


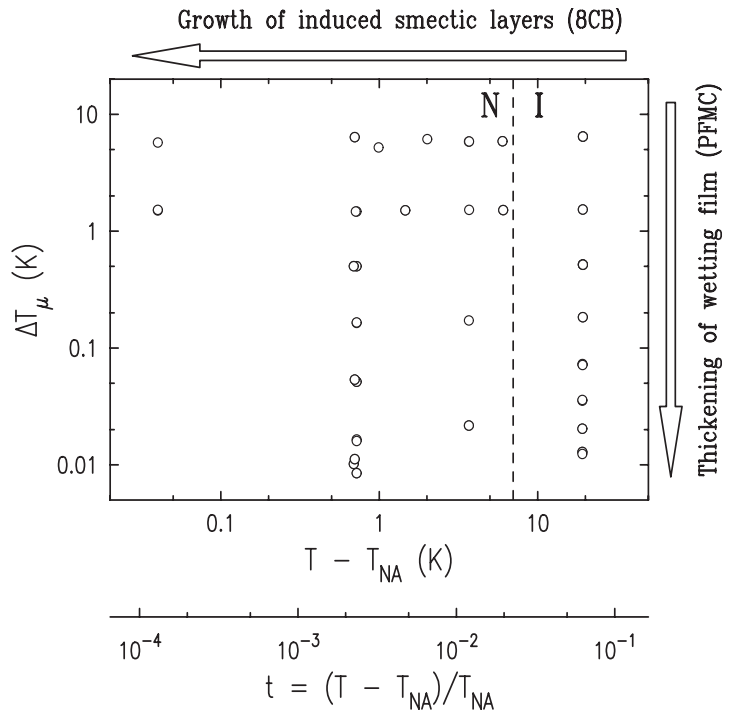

FIG. 2. Temperature settings at which XR measurements were made on PFMC wetting films on $8 \mathrm{CB}$ (open circles). The abscissa $T-T_{\mathrm{NA}}$ is the sample temperature relative to the NA transition of $8 \mathrm{CB}$ and $t=T / T_{\mathrm{NA}}-1$ is the associated reduced temperature. The ordinate $\Delta T_{\mu}=T-T_{\text {res }}$ is the temperature offset between the sample and the PFMC reservoir. The vertical dashed line indicates the transition temperature $T_{\mathrm{NI}}$ between the nematic $(\mathrm{N})$ and isotropic (I) phases of $8 \mathrm{CB}$.

was spread over the Pyrex disk using a spatula. This resulted in a puddle of liquid (isotropic) $8 \mathrm{CB}$, approximately $40 \mathrm{~mm}$ in diameter. The cell was then sealed without PFMC and the entire chamber was mounted on the liquid surface spectrometer. The XR measurements were first carried out on the free surface of pure $8 \mathrm{CB}$.

In order to produce saturated vapor needed to form a wetting film, a reservoir of PFMC was introduced, as described previously [24], by injecting $10 \mathrm{ml}$ of PFMC to the cell bottom. After setting the temperatures, the sample was left undisturbed until the film temperature $T$ and offset $\Delta T_{\mu}$ stabilized, typically for $1-3 \mathrm{~h}$, prior to starting $\mathrm{x}$-ray measurements. At each setting of $\left(T, \Delta T_{\mu}\right)$, indicated in Fig. 2, two or more XR scans were taken to ensure that the wetting film was stable.

The nematic-smectic- $A$ (NA) transition temperature $T_{\mathrm{NA}}$ is known to drift slowly with time [11,37-39]. Therefore, $T_{\mathrm{NA}}$ was checked periodically by heating and cooling the sample across $T_{\mathrm{NA}}$ (typically at $1-10 \mathrm{mK} / \mathrm{min}$ ) and monitoring the diffracted $x$-ray intensity of the first Bragg peak at $q_{z}=q_{0}=2 \pi / d=0.20 \AA^{-1}$, where the smectic layer spacing is $d=31.7 \AA$ for $8 \mathrm{CB}[32,37,40]$. The variations in $T_{\mathrm{NA}}$, determined by the onset of a significant intensity increase upon cooling or the termination of an intensity drop upon heating, were $33.31<T_{\mathrm{NA}}<33.47{ }^{\circ} \mathrm{C}$ (over $36 \mathrm{~h}$ ) and $33.45<T_{\mathrm{NA}}$ $<33.48{ }^{\circ} \mathrm{C}(16 \mathrm{~h})$ for two preparations of pure $8 \mathrm{CB}$, and $33.73<T_{\mathrm{NA}}<33.77{ }^{\circ} \mathrm{C}(40 \mathrm{~h})$ and $33.32<T_{\mathrm{NA}}<33.33{ }^{\circ} \mathrm{C}$ $(26 \mathrm{~h})$ for two preparations of PFMC-coated $8 \mathrm{CB}$. These values agree with the published values of $T_{\mathrm{NA}}$ $=33.0-33.9{ }^{\circ} \mathrm{C}$ for $8 \mathrm{CB}$ (e.g., $[13,31-35,37,40]$ ).
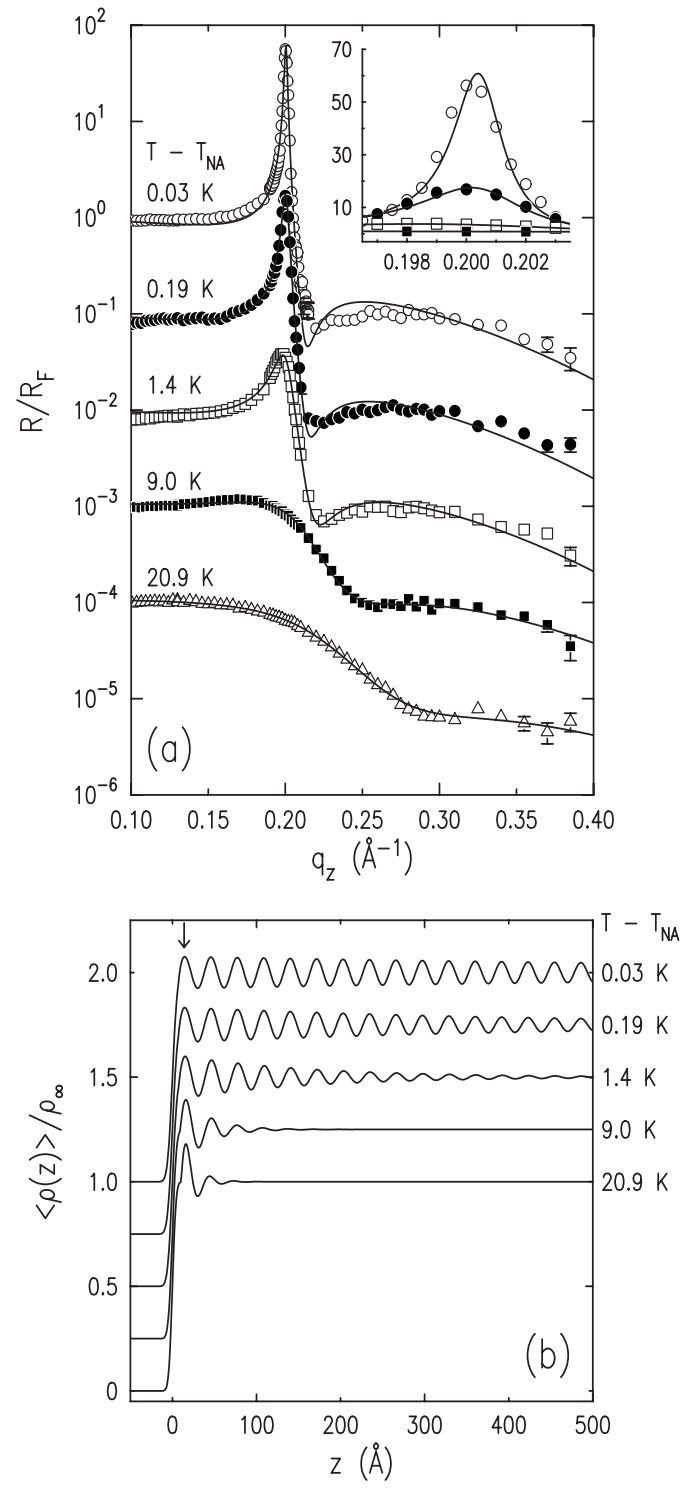

FIG. 3. (a) Measured Fresnel-normalized specular reflectivity $R / R_{F}$ vs $q_{z}$ (symbols), obtained from the free surface of bare 8CB at $T-T_{\mathrm{NA}}=0.03,0.19,1.4,9.0$, and $20.9 \mathrm{~K}$ (top to bottom), shifted vertically for clarity. The inset shows the linear plot of the same data near the quasi-Bragg peak at $q_{z}=q_{0}=0.2 \AA^{-1}$. The solid line through each data set represents the best fit from the XR analysis. These XR curves are consistent with the published results $[7,10]$ for the free surface of nematic $8 \mathrm{OCB}$, an analog of $8 \mathrm{CB}$. (b) The average electron-density profiles $\langle\rho(z)\rangle / \rho_{\infty}$ for the $8 \mathrm{CB}$-vapor interface, corresponding to the best-fit $R / R_{F}$ curves in (a), shifted vertically for clarity. The downward arrow in (b) indicates the position $z_{1}=z_{0}+d / 4$ of the central plane of the first smectic layer.

\section{RESULTS AND DISCUSSION}

\section{A. XR results, analysis, and electron density profiles}

Figure 3(a) shows representative normalized XR data (symbols) for the bare surface of $8 \mathrm{CB}$ (i.e., no PFMC). The data for PFMC wetting films on $8 \mathrm{CB}$ at different values of $\Delta T_{\mu}$ are summarized in Figs. 4(a) -4 (c) for $T-T_{\mathrm{NA}}=0.7,3.7$, and $19.3 \mathrm{~K}$, respectively. The XR data are shown in terms of the normalized reflectivity $R\left(q_{z}\right) / R_{F}\left(q_{z}\right)$, where $R_{F}\left(q_{z}\right)$ is the 

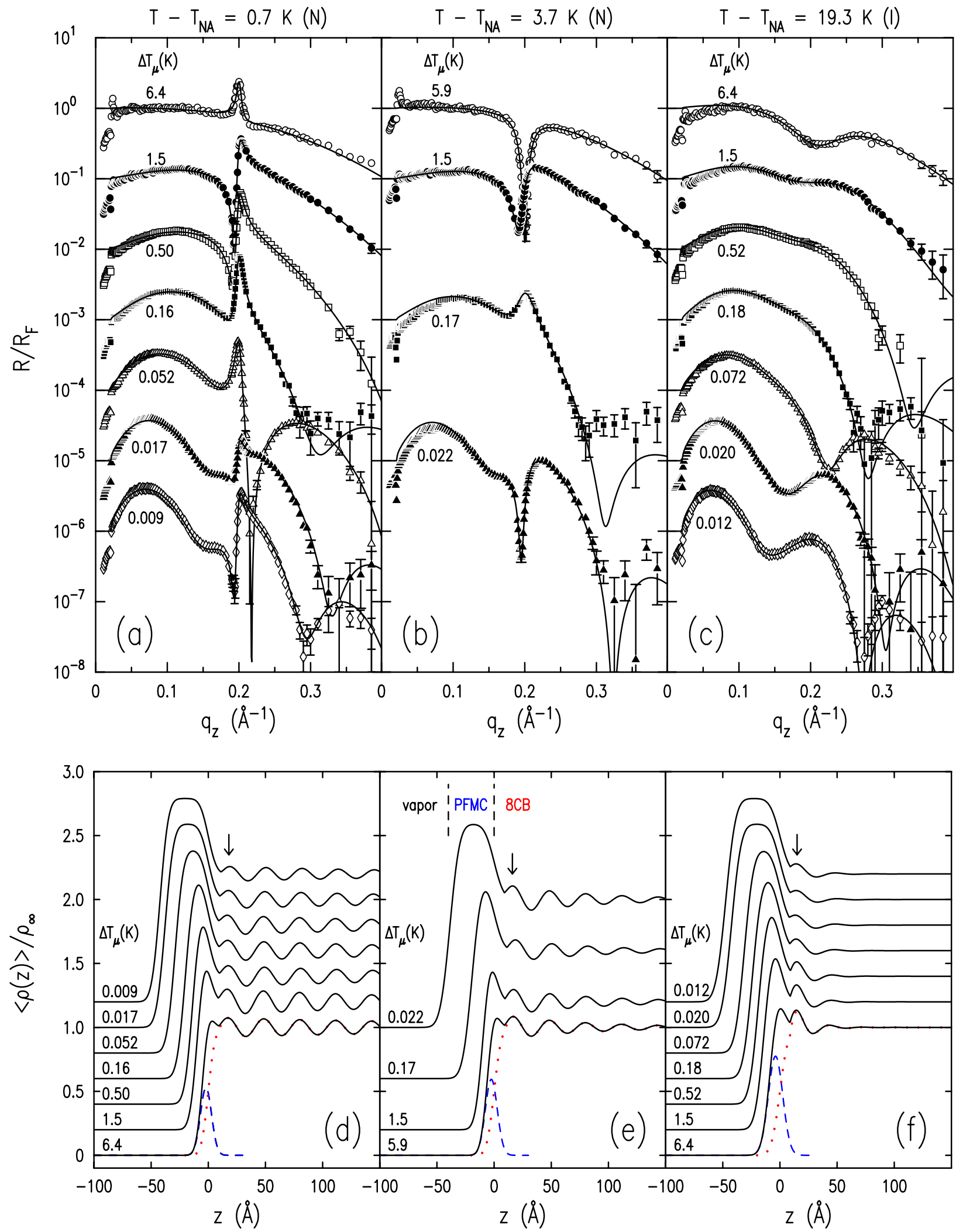

FIG. 4. (Color online) (a)-(c) Measured Fresnel-normalized reflectivity $R / R_{F}$ vs $q_{z}$ (symbols), obtained from PFMC-coated surfaces of $8 \mathrm{CB}$ at various $\Delta T_{\mu}$ for $T-T_{\mathrm{NA}}=$ (a) 0.7 , (b) 3.7, and (c) $19.3 \mathrm{~K}$. The solid line through each data set represents the best fit from the XR analysis. (d)-(f) The average electron-density profiles $\langle\rho(z)\rangle / \rho_{\infty}$ (solid lines) corresponding to the best-fit $R / R_{F}$ curves in (a)-(c), respectively. In (a)-(f), curves for different $\Delta T_{\mu}$ are shifted vertically for clarity. In (d)-(f), the PFMC and $8 \mathrm{CB}$ contributions to $\langle\rho(z)\rangle / \rho_{\infty}$ for the highest $\Delta T_{\mu}$ (bottommost) are indicated by the dashed and dotted lines, respectively, and the downward arrows (above the topmost profiles) indicate the position $z_{1}=z_{0}+d / 4$ of the central plane of the first smectic layer. 


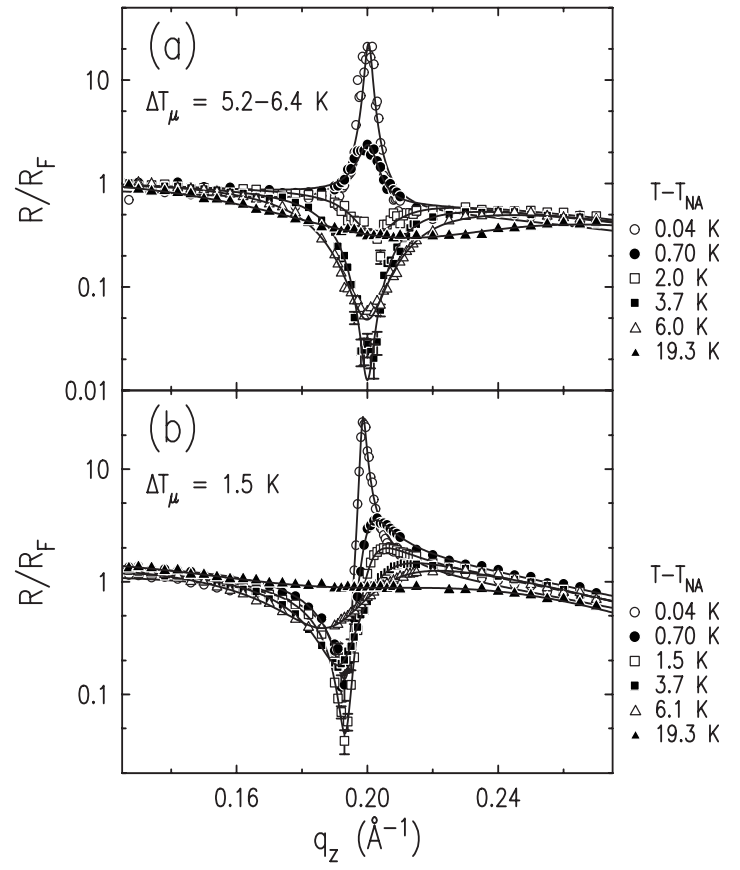

FIG. 5. Measured $R\left(q_{z}\right) / R_{F}\left(q_{z}\right)$ from PFMC-coated 8CB (symbols), emphasizing the $\left(T-T_{\mathrm{NA}}\right)$-dependent variation of the feature at $q_{z}=0.2 \AA^{-1}$ for $\Delta T_{\mu}=$ (a) $5.2-6.4 \mathrm{~K}$ and (b) $1.5 \mathrm{~K}$. The solid line through each data set represents the best fit from the XR analysis.

Fresnel reflectivity [41] that would be obtained for an ideally sharp and flat interface for which the electron density changes discontinuously from zero on the vapor side to the bulk average value $\rho_{\infty}\left(=\rho_{8 \mathrm{CB}}\right)$ of the subphase (i.e., $\left.8 \mathrm{CB}\right)$. The factor $R_{F}$, which depends on the critical wave vector $q_{c}=4 \sqrt{\pi r_{0} \rho_{\infty}}$ for total reflection with $r_{0}$ denoting the classical electron radius [41], was calculated by using the $T$-dependent bulk electron density $\rho_{\infty}(T)$ based on the mass density of 8CB [33]. The estimated value of $q_{c}$ is $0.0214 \AA^{-1}$ just above $T_{\mathrm{NA}}$ and decreases with increasing $T$ to $0.0211 \AA^{-1}$ at the highest probed temperature of $T=53{ }^{\circ} \mathrm{C}>T_{\mathrm{NI}}$.

As shown in Fig. 3(a), the XR data from the bare 8CBvapor interface display a peak at $q_{z}=q_{0} \approx 0.20 \AA^{-1}$ that grows sharper and larger in amplitude as $T-T_{\mathrm{NA}} \rightarrow 0^{+}$. This behavior, similar to the previous observations at the free surface of nematic 4-octyloxy-4'-cyanobiphenyl (8OCB) $[7,10,11]$, is consistent with the growth of surface-induced smectic layers with a $d$ spacing of $d=2 \pi / q_{0} \approx 31 \AA$.

The presence of a PFMC layer, by contrast, makes the $q_{z}$ dependence of the XR curves much more complicated, as shown in Figs. 4(a)-4(c). Qualitatively, the observed $R / R_{F}$ interference patterns can be seen as a superposition of two main features. The first feature is centered at $q_{0} \approx 0.20 \AA^{-1}$, has a shape and a sign that depend on $\Delta T_{\mu}$, and becomes sharper in $q_{z}$ as $T-T_{\mathrm{NA}} \rightarrow 0^{+}$. These characteristics are more clearly evident in Fig. 5, showing the $T-T_{\mathrm{NA}}$ dependence of $R / R_{F}$ data from the 8CB-PFMC-vapor interface at two different values of $\Delta T_{\mu}$. Note that the feature at $q_{0}$ is a peak or an antipeak for $\Delta T_{\mu}=6 \mathrm{~K}$ [Fig. 5(a)], whereas it is a dip followed by a peak for $\Delta T_{\mu}=1.5 \mathrm{~K}$ [Fig. 5(b)]; in both cases, the feature sharpens as $T$ approaches $T_{\mathrm{NA}}$. The second feature consists of an oscillation in the amplitude of $R / R_{F}$
(Kiessig fringes) whose period in $q_{z}$ decreases as $\Delta T_{\mu} \rightarrow 0^{+}$. If the feature at $q_{0}$ were absent, the $R / R_{F}$ data over $0 \leq q_{z}$ $\leq 0.4 \AA^{-1}$ [Figs. 4(a)-4(c)] would contain one oscillation maximum for $0.5 \leq \Delta T_{\mu} \leq 6 \mathrm{~K}$, two maxima for $\Delta T_{\mu}$ $\approx 0.05 \mathrm{~K}$, and three maxima for $\Delta T_{\mu} \approx 0.01 \mathrm{~K}$, regardless of $T-T_{\mathrm{NA}}$. This behavior is consistent with the presence of a wetting layer whose thickness increases with decreasing $\Delta T_{\mu}$.

The average electron density profile $\langle\rho(z)\rangle$ along the surface normal has been extracted by fitting model reflectivity curves to the data. The theoretical reflectivity was calculated using [42]

$$
\frac{R\left(q_{z}\right)}{R_{F}\left(q_{z}\right)}=\left|\int d z \frac{d}{d z}\left(\frac{\langle\rho(z)\rangle}{\rho_{\infty}}\right) e^{i \sqrt{q_{z} q_{z^{2}}}}\right|^{2},
$$

where $q_{t} \equiv \sqrt{q_{z}^{2}-q_{c}^{2}}$. Equation (1) reduces to the well-known Born approximation (BA) expression [41] at large $q_{z} \gg q_{c}$. However, at small $q_{z}$, Eq. (1) is equivalent to the low- $q_{z}$ limit of the form based on the distorted-wave Born approximation (DWBA) for a single fluctuating interface [42], and therefore provides a more accurate description of $R\left(q_{z}\right)$ than the BA.

The model profile that was used to describe the $8 \mathrm{CB}$ PFMC-vapor interface is given by

$$
\begin{aligned}
\frac{\langle\rho(z)\rangle}{\rho_{\infty}}= & \phi_{1}(z)+\phi_{2}(z)+\phi_{3}(z) \\
= & \frac{1}{2}\left[1+\operatorname{erf}\left(\frac{z}{\sqrt{2} \sigma}\right)\right] \\
& +\Theta\left(z-z_{0}\right) A e^{-\left(z-z_{0}\right) / \xi} \sin \left[2 \pi\left(z-z_{0}\right) / d\right] \\
& +\frac{\phi_{L}}{2}\left[\operatorname{erf}\left(\frac{z+L}{\sqrt{2} \sigma}\right)-\operatorname{erf}\left(\frac{z}{\sqrt{2} \sigma}\right)\right],
\end{aligned}
$$

where the Heaviside step function $\Theta\left(z-z_{0}\right)$ is equal to unity for $z \geq z_{0}$ and zero for $z<z_{0}$. The first two terms $\phi_{1}+\phi_{2}$ are identical to the model used previously $[10,11]$ to describe the induced smectic layering at the vapor interface. The errorfunction term $\phi_{1}$ would describe the subphase-vapor interface in the absence of an adsorbed film. The exponentially decaying sine term $\phi_{2}$ accounts for the induced smectic layers, where $d$ is the smectic layer spacing, $\xi$ is the decay length, and $A$ is the amplitude of the density oscillation at $z=z_{0}$. The third term $\phi_{3}$, representing an error-function-based "box" layer, accounts for the presence of the PFMC wetting layer, where $L$ is the layer thickness and $\phi_{L}$ is the layer's electron density relative to the bulk subphase. In order to keep the model as simple as possible, the roughnesses of the 8CB-PFMC and PFMC-vapor interfaces have been set to be equal, so that a single roughness parameter $\sigma$ is used in all the error functions in Eq. (2). Allowing the roughness of the two interfaces to vary independently did not significantly improve the fits.

Substituting Eq. (2) in Eq. (1), the resulting analytical expression for $R / R_{F}$ has been used to calculate the model reflectivity curves. For the XR data from a pure $8 \mathrm{CB}$ surface (i.e., in the absence of PFMC), five parameters $A, d, \xi, z_{0}$, and $\sigma$ were varied in the fitting, with $\phi_{L}=0$. For the PFMCcoated $8 \mathrm{CB}$ surfaces, six parameters $A, d, \xi, z_{0}, \sigma$, and $L$ 
TABLE I. List of best-fit parameters for fitting to the XR data for the free surface of bare 8CB in Fig. 3 . The electron density profile $\langle\rho(z)\rangle / \rho_{\infty}$ was modeled using Eq. (2) with $\phi_{L}=0$.

\begin{tabular}{cccccc}
\hline \hline $\begin{array}{c}T-T_{\mathrm{NA}} \\
(\mathrm{K})\end{array}$ & $A$ & $\begin{array}{c}d \\
(\AA)\end{array}$ & $\begin{array}{c}\xi \\
(\AA)\end{array}$ & $\begin{array}{c}z_{0} \\
(\AA)\end{array}$ & $\begin{array}{c}\sigma \\
(\AA)\end{array}$ \\
\hline 0.03 & $0.077 \pm 0.002$ & $31.43 \pm 0.01$ & $981 \pm 59$ & $6.5 \pm 0.7$ & $4.5 \pm 0.1$ \\
0.19 & $0.085 \pm 0.002$ & $31.43 \pm 0.02$ & $463 \pm 25$ & $6.7 \pm 0.5$ & $4.5 \pm 0.1$ \\
1.44 & $0.105 \pm 0.004$ & $31.44 \pm 0.05$ & $155 \pm 9$ & $7.4 \pm 0.3$ & $4.4 \pm 0.1$ \\
9.01 & $0.180 \pm 0.013$ & $30.3 \pm 0.5$ & $31 \pm 2$ & $9.2 \pm 0.6$ & $3.8 \pm 0.3$ \\
20.9 & $0.28_{-0.07}^{+0.11}$ & $28.3_{-1.1}^{+1.7}$ & $15 \pm 3$ & $10.2_{-1.4}^{+0.7}$ & $3.4 \pm 0.7$ \\
\hline \hline
\end{tabular}

were varied. In order to minimize the number of free parameters, the relative density $\phi_{L}$ of the wetting layer was kept fixed at the physically expected value of $\phi_{L}=\rho_{\mathrm{PFMC}} / \rho_{8 \mathrm{CB}}$ $=1.59$ [43], which is approximately independent of $T$ over the probed range of $T_{\mathrm{NA}}<T<53{ }^{\circ} \mathrm{C}$. For most of the XR data, the data points for $q_{z} \geq 0.1 \AA^{-1}$ were included in the fits. The only exception is that, for the data sets from the PFMC films with the largest probed thickness $(L>40 \AA)$, for which $R / R_{F}$ rises steeply just above $q_{c}$, the fitting range of $q_{z} \geq 0.06 \AA^{-1}$ was used instead. The best-fit $R / R_{F}$ curves for bare and PFMC-coated 8CB surfaces are shown as solid lines in Fig. 3(a) and in Figs. 4(a)-4(c) and 5, respectively, and the corresponding best-fit electron density profiles $\langle\rho(z)\rangle / \rho_{\infty}$ are shown in Figs. 3(b) and 4(d)-4(f). The best-fit parameters for the shown curves are listed in Table I (bare
8CB) and in Table II (PFMC films on 8CB).

The extracted profiles clearly demonstrate both the presence of interface-induced smectic layers on the liquid-crystal side and their growth as $T-T_{\mathrm{NA}} \rightarrow 0^{+}$. Regardless of whether the liquid crystal is in contact with vapor [Fig. 3(b)] or with a liquid (PFMC) film [Figs. 4(d)-4(f)], the 8CB side of $\langle\rho(z)\rangle / \rho_{\infty}$ exhibits an oscillation whose periodicity is essentially equal to the smectic layer spacing of $d=31.7 \AA$ for $8 \mathrm{CB}[32,37,40]$, and the effective number of induced smectic layers, as measured by $\xi$, increases with decreasing $T-T_{\mathrm{NA}}$. The profiles further indicate that, at each $T-T_{\mathrm{NA}}$, the wetting film thickness grows monotonically as $\Delta T_{\mu} \rightarrow 0^{+}$[Figs. 4(d)-4(f); Table II]. Together with the apparent insensitivity of $T_{\mathrm{NA}}$ to the presence of PFMC (Sec. II B), the fact that the XR data can be fitted well with the expected value of $\phi_{L}$

TABLE II. List of best-fit parameters for fitting to the XR data for PFMC-coated surfaces of 8CB in Fig. 4. The electron density profile $\langle\rho(z)\rangle / \rho_{\infty}$ was modeled using Eq. (2) with $\phi_{L}=1.59$.

\begin{tabular}{|c|c|c|c|c|c|c|c|}
\hline $\begin{array}{c}T-T_{\mathrm{NA}} \\
(\mathrm{K})\end{array}$ & $\begin{array}{l}\Delta T_{\mu} \\
(\mathrm{K})\end{array}$ & $A$ & $\begin{array}{c}d \\
(\AA)\end{array}$ & $\begin{array}{c}\xi \\
(\AA)\end{array}$ & $\begin{array}{c}z_{0} \\
(\AA)\end{array}$ & $\begin{array}{c}\sigma \\
(\AA)\end{array}$ & $\begin{array}{c}L \\
(\AA)\end{array}$ \\
\hline \multicolumn{8}{|c|}{ (a) $T-T_{\mathrm{NA}}=0.7 \mathrm{~K}$} \\
\hline 0.70 & 6.38 & $0.076 \pm 0.015$ & $31.42 \pm 0.17$ & $305 \pm 78$ & $9.3 \pm 0.9$ & $4.7 \pm 0.4$ & $4.1 \pm 0.8$ \\
\hline 0.71 & 1.47 & $0.102 \pm 0.013$ & $31.58 \pm 0.07$ & $216 \pm 24$ & $8.8 \pm 0.5$ & $5.3 \pm 0.1$ & $9.1 \pm 0.3$ \\
\hline 0.72 & 0.502 & $0.085 \pm 0.005$ & $31.56 \pm 0.05$ & $221 \pm 18$ & $9.8 \pm 0.4$ & $5.6 \pm 0.1$ & $13.6 \pm 0.3$ \\
\hline 0.72 & 0.164 & $0.082 \pm 0.005$ & $31.52 \pm 0.05$ & $230 \pm 19$ & $9.3 \pm 0.5$ & $5.8 \pm 0.1$ & $20.1 \pm 0.4$ \\
\hline 0.72 & 0.052 & $0.079 \pm 0.005$ & $31.48 \pm 0.06$ & $230 \pm 21$ & $9.0 \pm 0.7$ & $5.8 \pm 0.1$ & $29.0 \pm 0.3$ \\
\hline 0.72 & 0.016 & $0.072 \pm 0.010$ & $31.65 \pm 0.25$ & $259 \pm 53$ & $10.0 \pm 0.5$ & $6.0 \pm 0.1$ & $37.8 \pm 0.4$ \\
\hline 0.72 & 0.009 & $0.059 \pm 0.006$ & $31.57 \pm 0.10$ & $221 \pm 32$ & $11.1 \pm 0.7$ & $5.8 \pm 0.1$ & $42.2 \pm 0.4$ \\
\hline \multicolumn{8}{|c|}{ (b) $T-T_{\mathrm{NA}}=3.7 \mathrm{~K}$} \\
\hline 3.69 & 5.86 & $0.094_{-0.009}^{+0.046}$ & $31.54 \pm 0.24$ & $76 \pm 7$ & $9.2 \pm 0.7$ & $5.0 \pm 0.3$ & $4.9 \pm 0.8$ \\
\hline 3.69 & 1.52 & $0.106 \pm 0.008$ & $31.62 \pm 0.12$ & $76 \pm 5$ & $9.5 \pm 0.3$ & $5.5 \pm 0.1$ & $9.3 \pm 0.4$ \\
\hline 3.68 & 0.172 & $0.092 \pm 0.009$ & $31.55 \pm 0.17$ & $74 \pm 7$ & $11.3 \pm 0.7$ & $6.5 \pm 0.1$ & $19.3 \pm 0.3$ \\
\hline 3.68 & 0.022 & $0.112 \pm 0.014$ & $31.72 \pm 0.22$ & $78 \pm 8$ & $9.0 \pm 0.4$ & $6.7 \pm 0.2$ & $38.6 \pm 0.3$ \\
\hline \multicolumn{8}{|c|}{ (c) $T-T_{\mathrm{NA}}=19.3 \mathrm{~K}$} \\
\hline 19.3 & 6.45 & $0.21_{-0.05}^{+0.07}$ & $29 \pm 4$ & $14 \pm 4$ & $8.8 \pm 0.6$ & $5.9 \pm 1.0$ & $8 \pm 3$ \\
\hline 19.3 & 1.53 & $0.21_{-0.11}^{+0.23}$ & $30 \pm 7$ & $13_{-5}^{+8}$ & $9.1 \pm 1.3$ & $6.2_{-0.5}^{+0.9}$ & $14 \pm 4$ \\
\hline 19.3 & 0.519 & $0.18_{-0.11}^{+0.29}$ & $30 \pm 6$ & $13_{-6}^{+10}$ & $9.2 \pm 1.6$ & $6.3_{-0.2}^{+0.4}$ & $19 \pm 3$ \\
\hline 19.3 & 0.184 & $0.14_{-0.10}^{+0.18}$ & $33_{-6}^{+9}$ & $14_{-7}^{+16}$ & $9 \pm 3$ & $6.5 \pm 0.4$ & $24 \pm 2$ \\
\hline 19.2 & 0.072 & $0.11_{-0.05}^{+0.11}$ & $33_{-4}^{+5}$ & $16_{-7}^{+9}$ & $8.2 \pm 1.1$ & $6.5 \pm 0.1$ & $31.2 \pm 0.8$ \\
\hline 19.1 & 0.020 & $0.10_{-0.05}^{+0.11}$ & $34_{-3}^{+5}$ & $15_{-7}^{+10}$ & $8.9 \pm 1.0$ & $6.7 \pm 0.1$ & $42.4 \pm 0.8$ \\
\hline 19.1 & 0.012 & $0.11_{-0.05}^{+0.12}$ & $33_{-4}^{+5}$ & $15_{-7}^{+10}$ & $9.0 \pm 1.0$ & $6.9 \pm 0.1$ & $47.4 \pm 0.9$ \\
\hline
\end{tabular}


$=1.59$ supports the assumption about the immiscibility between PFMC and 8CB.

The XR data analysis above provides quantitative insights into the induced smectic layering of $8 \mathrm{CB}$ (through $d, \xi, A$, and $z_{0}$ ) and the wetting behavior of PFMC (via $L$ ). These two phenomena are discussed separately in Secs. III B and III C below. We conclude this section with comments on the extracted interfacial roughness $\sigma$.

For a single fluid-fluid interface, the capillary-wave contribution $\sigma_{c}$ to the measured roughness is often well approximated by $\sigma_{c}^{2}=\left(k_{B} T / 2 \pi \gamma\right) \ln \left(q_{\max } / \Delta q_{y}\right)[24,44]$. The surface tension is $\gamma=26 \mathrm{mN} / \mathrm{m}$ for $8 \mathrm{CB}$ just above $T_{\mathrm{NA}}$ [31], the upper cutoff wave vector for the shortest allowed capillary wavelength is estimated to be $q_{\max }=0.5 \AA^{-1}$ [45], and the full width at half maximum (FWHM) detector resolution (Sec. II A) can be taken to be $\Delta q_{y}=0.0004 \AA^{-1}$ at the midpoint $q_{z}=0.3 \AA^{-1}$ of the typical fitting range. These values yield an estimated capillary roughness of $\sigma_{c}=4.4 \AA$, in good agreement with the extracted values of $\sigma$ for the 8CB-vapor interface (Table I). This demonstrates that the width of the bare $8 \mathrm{CB}$ surface is dominated by the capillary roughness and that the intrinsic width is negligible. As for the PFMCcoated $8 \mathrm{CB}$ surface, the interpretation of $\sigma$ is somewhat ambiguous since the use of this single roughness parameter neglects the fact that the widths of the two interfaces (8CBPFMC and PFMC-vapor) are expected to be different $[24,27]$. Nevertheless, it is physically reasonable that wetting, which lowers the interfacial energy, results in larger values of $\sigma$ for the PFMC-coated 8CB (Table II) than those for the 8CB-vapor interface. Moreover, at each fixed temperature $T-T_{\mathrm{NA}}$, the extracted $\sigma$ tends to increase with increasing PFMC thickness $L$. This behavior is qualitatively consistent with the decoupling of the two interfaces at large separation, which has been shown to result in an enhancement of the capillary roughness at each interface [24,27].

\section{B. Induced smectic layering at liquid-liquid crystal interfaces}

The temperature dependence of the fitted $\xi$, the surfaceinduced smectic decay length, is plotted in Fig. 6(a), both for the free surface of $8 \mathrm{CB}$ and for the PFMC-coated $8 \mathrm{CB}$ surface. For comparison, the solid line in the figure represents the longitudinal bulk correlation length $\xi_{\|}=\xi_{\|, 0}\left(T / T_{\mathrm{NA}}\right.$ $-1)^{-\nu_{\|}}=\left(\begin{array}{ll}3.8 \AA & \AA\end{array}\right)\left(T / T_{\mathrm{NA}}-1\right)^{-0.70}$ for the smectic order in the nematic phase of 8CB, based on Ref. [39] (also see Refs. $[37,40])$. The diverging $\xi_{\|}(T)$ is associated with the nearly second-order nature of the NA transition of $8 \mathrm{CB}[34,37,40]$ and signifies the enhancement of critical fluctuations as $T$ $\rightarrow T_{\mathrm{NA}}$.

Figure 6(a) shows that, independent of whether the $8 \mathrm{CB}$ surface is bare or coated with PFMC, the measured values of $\xi$ are in good agreement with $\xi_{\|}$in terms of both magnitudes and $T$ dependence, except at the smallest probed values of $T-T_{\mathrm{NA}}<0.1 \mathrm{~K}[46]$. The observation $\xi(T) \approx \xi_{\|}(T)$ implies that the induced smectic layering at both the 8CB-vapor (nematic-vapor) and 8CB-PFMC (nematic-liquid) interfaces is driven by critical adsorption, originating from (i) the existence of a boundary condition that favors the formation of
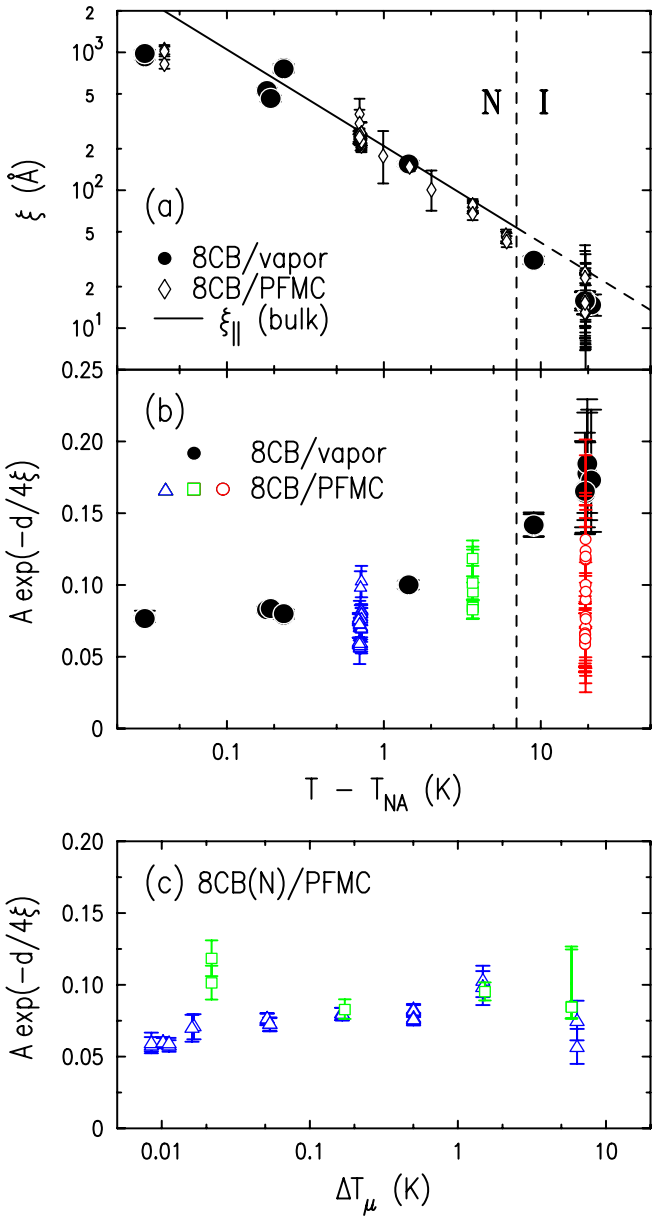

FIG. 6. (Color online) The extracted decay length $\xi$ of the density oscillation for the induced smectic layering and the oscillation amplitude $A_{1}=A \exp (-d / 4 \xi)$ [in units of $\langle\rho(z)\rangle / \rho_{\infty}$ ] at the center position $z_{1}=z_{0}+d / 4$ of the first smectic layer, obtained from the XR analysis. (a) $\xi$ and (b) $A_{1}$ as functions of $T-T_{\mathrm{NA}}$ for the $8 \mathrm{CB}$-vapor interface (filled circles) and the PFMC-coated 8CB (open symbols), where the vertical dashed line indicates $T_{\mathrm{NI}}$ for $8 \mathrm{CB}$. In (a), the solid line corresponds to the longitudinal bulk correlation length $\xi_{\|}=(3.8 \AA)\left(T / T_{\mathrm{NA}}-1\right)^{-0.70}$ for the smectic fluctuations in nematic 8CB [39], where its extrapolation beyond $T_{\mathrm{NI}}$ is shown as the dashed line. (c) $A_{1}$ vs $\Delta T_{\mu}$ for PFMC-coated nematic $8 \mathrm{CB}$ at $T$ $-T_{\mathrm{NA}}=0.7 \mathrm{~K}$ (open triangles) and $3.7 \mathrm{~K}$ (open squares).

the first smectic layer and (ii) the presence of critical fluctuations that leads to the persistence of layering into the bulk material. A similar $T$-dependent smectic growth behavior has been observed previously at the free surface of an $8 \mathrm{CB}$ ana$\log , 8 \mathrm{OCB}[6,7,10,11]$, and at buried interfaces between nematic $8 \mathrm{CB}$ and solid substrates $[8,13]$.

The effects of vapor and PFMC on the boundary conditions of $8 \mathrm{CB}$ can be examined further by comparing the amplitude $A_{1} \equiv A \exp (-d / 4 \xi) \approx\left\langle\rho\left(z_{1}\right)\right\rangle / \rho_{\infty}-1$ of the smectic density oscillation at the maximum position $z_{1} \equiv z_{0}+d / 4$, corresponding to the middle of the first smectic layer [Figs. 3(b) and 4(d)-4(f)]. The amplitude $A_{1}$ should serve as a reliable measure of the surface smectic order parameter $\psi_{s}$ in the case of nematic $8 \mathrm{CB}$, for which the induced smectic lay- 
ers are well defined and extend far from the interface. The extracted $A_{1}$, based on the best-fit parameters $(A, d, \xi)$, is plotted as a function of $T-T_{\mathrm{NA}}$ and $\Delta T_{\mu}$ in Figs. 6(b) and 6(c), respectively. On the nematic side $\left(T-T_{\mathrm{NA}}<7 \mathrm{~K}\right)$ in Fig. 6(b), the $A_{1}$ values for the PFMC-coated 8CB are comparable to those obtained for bare $8 \mathrm{CB}$, within the scatter of the data. For the PFMC-coated nematic 8CB surface, Fig. 6(c) further shows no systematic dependence of $A_{1}$ on $\Delta T_{\mu}$. These observations strongly suggest that the surface order parameter $\psi_{s}$ is independent of whether the nematic 8CB is in contact with vapor or PFMC and remains unchanged no matter how much PFMC liquid is adsorbed, at least up to the maximum probed PFMC thickness of $L \sim 50 \AA$. This result should be contrasted from the recent study by Lau et al. [13] of the smectic layer growth at nematic-8CB-solid-substrate interfaces, in which the surface order parameter was found to differ, by as much as a factor of 2, among four different surface treatments of $\mathrm{Si} / \mathrm{SiO}_{2}$ substrates.

In the smectic- $A$ phase of $8 \mathrm{CB}$, each smectic layer consists of a bilayerlike molecular arrangement such that the cyanobiphenyl head groups segregate toward the central plane of the layer with some degree of overlap and the alkyl tails point away from the central plane, respectively [32]. Thus, the maxima of the density oscillation in the profiles $\langle\rho(z)\rangle$ [Figs. 3(b) and 4(d)-4(f)] arise from the segregation of the cyanobiphenyl groups, whereas the minima correspond to the regions rich in the terminal $-\mathrm{CH}_{3}$ groups. In view of this and Eq. (2), it is significant that the observed offset $z_{0}$ roughly satisfies $z_{0} \approx d / 4(\approx 8 \AA$; see Tables I and II $)$, implying that for both the 8CB-vapor and 8CB-PFMC interfaces the terminal methyl group of $8 \mathrm{CB}$ is at the interface $(z=0)$. This is consistent with the homeotropic (normal-tointerface) orientation of $8 \mathrm{CB}$ molecules at both these interfaces.

It should be noted that the present study provides a more definitive measure of the phase factor $z_{0}$ than the previous experiments on other liquid-crystal interfaces $[7,9,10,13]$. Tables I and II further indicate that the extracted values of $z_{0}$ for the 8CB-PFMC interface exhibit less scatter than those for the 8CB-vapor interface. This illustrates one advantage of having a thin, high-contrast overlayer (PFMC), which makes it possible to obtain reliable phase information on the induced smectic order by exploiting the interference between the scattering from the additional (PFMC-vapor) interface and the underlying density modulation.

The homeotropic orientation of $8 \mathrm{CB}$ at the vapor interface is consistent with the general observation that, for the same number of carbon atoms, $n$-alkanes exhibit lower surface tensions than aromatic compounds, and that the - $\mathrm{CH}_{3}$-terminated surface generally has a lower surface energy than those materials that expose the $-\mathrm{CH}_{2}$ - groups in the alkyl chain [47]. For example, the surface tension at $20{ }^{\circ} \mathrm{C}$ is: $\gamma=18.4 \mathrm{mN} / \mathrm{m}$ for $n$-hexane $\mathrm{CH}_{3}\left(\mathrm{CH}_{2}\right)_{4} \mathrm{CH}_{3}, 25.0 \mathrm{mN} / \mathrm{m}$ for cyclohexane $\left(\mathrm{CH}_{2}\right)_{6}$, and $28.9 \mathrm{mN} / \mathrm{m}$ for benzene $\mathrm{C}_{6} \mathrm{H}_{6}$. Similarly, the observation of the homeotropically aligned $8 \mathrm{CB}$ at the interface with PFMC suggests that the minimization of overall interfacial energy favors the terminal $-\mathrm{CH}_{3}$ groups rather than the $-\mathrm{CH}_{2}$ - or cyanobiphenyl groups to be in contact with the fluorocarbon. This is one of the main results of the present study and is relevant, e.g., to the increasingly active research on the alignment of the columnar phases of discotic liquid crystals using fluorocarbon surfaces $[14,15]$. Not unlike $8 \mathrm{CB}$, these diskotic molecules are often composed of an aromatic core and emanating alkyl side chains.

The robustness of induced smectic layering at the PFMC$8 \mathrm{CB}$ interface is to be contrasted with our previous study of PFMC-coated surfaces of bulk liquid eicosane (C20), an $n$-alkane $[23,24]$. That study showed that an ultrathin layer of PFMC ( $L \sim 7 \AA$ A) was sufficient to completely suppress the surface frozen phase of C20 [23]. The very different effects of a PFMC thin film on the surface ordering behavior of $8 \mathrm{CB}$ and $\mathrm{C} 20$ reflect the fundamentally different nature of surfaceinduced smectic layering and the surface freezing phenomenon. Unlike the smectic- $A$ layers, which consist of a stacked two-dimensional (2D) liquid, the surface frozen monolayer of $n$-alkanes possesses a quasi-long-range $2 \mathrm{D}$ positional order. In the case of surface freezing at the air interface [5], the terminal methyl groups are believed to play the role of a surfactant since the $-\mathrm{CH}_{3}$ groups have a lower surface energy than the $-\mathrm{CH}_{2}$ - groups [47]. In turn, this promotes a conformational transition of the alkane chains to the all-trans configuration at a temperature where the underlying bulk remains molten [5]. However, at the PFMC interface, the absence of surface freezing suggests that the adsorption of $-\mathrm{CH}_{3}$ groups does not significantly lower the surface energy and the bulk and surface freeze at the same temperature. We also note that, at the solid interface, surface freezing of alkanes has never been observed [48]. On the other hand, in the nematic phase of a liquid crystal, the surface-induced smectic layering has the same persistence length as the bulk smectic fluctuations. This suggests that the surface plays the role of fixing the phase of the layering and does not affect the strength of the layering (i.e., $\xi$ and $A$ ). As with alkanes, the methyl groups are at the free surface. However, there is no change in the conformation of the chains associated with the NA transition. Thus, the driving force for the ordering is very different for these two systems. Despite these differences, however, the effect of the PFMC interface is apparently not strong enough to alter the orientational preference that favors the $-\mathrm{CH}_{3}$ groups at the interface.

\section{Wetting of liquid crystal surfaces}

The thickness $L$ of the PFMC wetting film on $8 \mathrm{CB}$, obtained from the best fits to the XR data, is plotted as a function of $\Delta T_{\mu}$ in Fig. 7 for the nematic 8CB subphase at $T$ $-T_{\mathrm{NA}}=0.7$ and $3.7 \mathrm{~K}$ and for the isotropic $8 \mathrm{CB}$ subphase at $T-T_{\mathrm{NA}}=19.3 \mathrm{~K}$ (open symbols). For further comparison, Fig. 7 also shows the previously measured data for the thickness of PFMC wetting films on bulk liquid eicosane (C20) at $37.2{ }^{\circ} \mathrm{C}$ (filled circles; from Ref. [23]).

A major finding of the present study is that the observed thickening behavior of PFMC with decreasing $\Delta T_{\mu}$ is consistent with complete wetting. In order to illustrate this, Fig. 7 shows calculated curves (solid and dashed lines) for the $1 / 3$ power law behavior $L=\left[A_{\text {eff }} /(6 \pi n \Delta \mu)\right]^{1 / 3} \propto\left(\Delta T_{\mu}\right)^{-1 / 3}$ that is expected for complete wetting films as the bulk liquid-vapor 


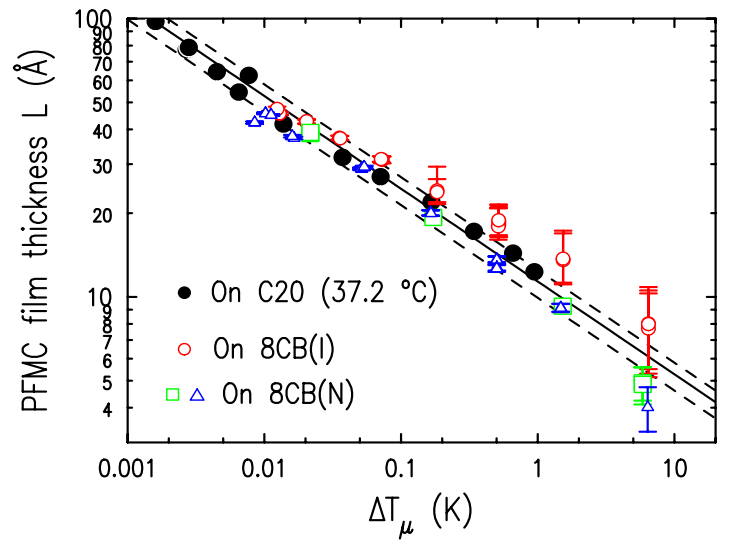

FIG. 7. (Color online) The extracted thickness $L$ of PFMC wetting films on $8 \mathrm{CB}$ as a function of $\Delta T_{\mu}$, obtained from the $\mathrm{XR}$ analysis, for PFMC on nematic $8 \mathrm{CB}$ at $T-T_{\mathrm{NA}}=0.7$ (open triangles) and $3.7 \mathrm{~K}$ (open squares) and on isotropic $8 \mathrm{CB}$ at $T-T_{\mathrm{NA}}$ $=19.3 \mathrm{~K}$ (open circles). The previously measured $L$ for PFMC films on the liquid eicosane $(\mathrm{C} 20)$ at $T=37.2{ }^{\circ} \mathrm{C}$ is shown as filled circles (from Ref. [23]). The lines represent the $1 / 3$ power law behavior $L \propto\left(\Delta T_{\mu}\right)^{-1 / 3}$ with an estimated effective Hamaker constant of $A_{\text {eff }}=(1.5 \pm 0.5) \times 10^{-20} \mathrm{~J}$ for PFMC on C20.

coexistence is approached, i.e., as $\Delta \mu \rightarrow 0^{+}$for the chemical potential offset $[1,2]$. These curves are based on (i) the approximation $\Delta \mu \approx(Q / T) \Delta T_{\mu}[26,27]$, (ii) the known latent heat of vaporization $Q$ and number density $n$ for liquid PFMC [49], and (iii) the effective Hamaker constant for the PFMC films on $\mathrm{C} 20$, which is estimated from this plot to be $A_{\text {eff }}=(1.5 \pm 0.5) \times 10^{-20} \mathrm{~J}$. For the PFMC films on $8 \mathrm{CB}$, similar graphical inspections yield an estimate of $A_{\text {eff }}$ $=(1.1 \pm 0.4) \times 10^{-20} \mathrm{~J}$ for the nematic $8 \mathrm{CB}$ and $(2.3 \pm 1.0)$ $\times 10^{-20} \mathrm{~J}$ for the isotropic $8 \mathrm{CB}$. Figure 7 shows that, within the uncertainties and scatter of the data, the observed $\Delta T_{\mu}$ dependence of $L$ can be described by the 1/3 power law at each $T-T_{\mathrm{NA}}$, especially well for the films on the nematic subphase.

The complete wetting behavior of PFMC is rather robust and largely unaffected by subtle variations in the nature of the subphase. First of all, the PFMC thickness observed for a given $\Delta T_{\mu}$ is not strongly dependent on $T-T_{\mathrm{NA}}$. Figure 7 shows that the thickness $L$ for the isotropic 8CB (open circles) tends to have slightly higher best-fit values than those for the nematic 8CB (open triangles and squares). However, for the thickest and thinnest films $(L>40 \AA$ or $L<10 \AA$ ), these differences in $L$ are no more than the associated error bars and scatter in the data. It can also be shown that a better agreement between the nematic and isotropic cases is obtained if a more sophisticated approach is used to extract the PFMC thickness from the XR-based density profiles [50]. Similarly, the $A_{\text {eff }}$ values estimated above for PFMC on nematic and isotropic 8CB agree with each other within their uncertainties. These observations imply that the amount of PFMC adsorbed on the 8CB surface is not strongly sensitive to the degree of interfacial smectic order, at least up to $\xi / d=7-10$ smectic layers at $T-T_{\mathrm{NA}}=0.7 \mathrm{~K}$ (Table II). This apparent insensitivity may be due to the fact that the van der Waals (vdW) interactions are long ranged and tend to smear out the effects of ordering at intermolecular length scales.

Second, both the magnitude and the $\Delta T_{\mu}$ dependence of $L$ for the PFMC films on $8 \mathrm{CB}$ agree well with those for the PFMC films on C20 (Fig. 7). Evidently, the presence of cyanobiphenyl groups in $8 \mathrm{CB}$ does not significantly alter the wetting behavior of PFMC as compared to the case of the alkane C20. This result indicates that, as generally expected [51], the vdW interactions between PFMC and 8CB are dominated by dispersion forces rather than by those involving the permanent dipole of the cyano group, the effect of which is reduced by the bilayerlike arrangement in the smectic layering.

The magnitudes of $A_{\text {eff }}$ estimated above are consistent with the vdW interactions between hydro- and fluorocarbons. With $A_{i j}$ denoting the Hamaker constant for nonretarded $\mathrm{vdW}$ interactions between media $i$ and $j, A_{\text {eff }}$ for a uniform fluorocarbon film ("F") on a uniform hydrocarbon subphase ("H") can be approximated as $A_{\mathrm{eff}} \approx A_{\mathrm{HF}}-A_{\mathrm{FF}} \approx \sqrt{A_{\mathrm{HH}} A_{\mathrm{FF}}}$ $-A_{\mathrm{FF}}[51]$. In the absence of hydrogen bonds, $A_{i i}$ can be estimated from the surface tension $\gamma_{i}$ of medium $i$ using the relation $A_{i i} / \gamma_{i} \approx 0.21 \times 10^{-20} \mathrm{~J} /(\mathrm{mN} / \mathrm{m})$ [Eq. (11.35) of Ref. [51]]. Taking $\gamma_{\mathrm{F}}=12.5 \mathrm{mN} / \mathrm{m}$ for PFMC [27] and $\gamma_{\mathrm{H}}$ $=26 \mathrm{mN} / \mathrm{m}$, which is within $1 \mathrm{mN} / \mathrm{m}$ of the known surface tensions for bare $8 \mathrm{CB}$ [31] and $\mathrm{C} 20$ [5] at relevant $T$, the above approximations yield $A_{\text {eff }} \approx 1.2 \times 10^{-20} \mathrm{~J}$, in good agreement with the $A_{\text {eff }}$ values estimated from the $\Delta T_{\mu}$ dependence of the PFMC thickness.

\section{SUMMARY}

We studied the interfacial structure of the liquid crystal 8CB which was coated by a thin wetting film of an immiscible liquid, the fluorocarbon PFMC. The electron density profile across the $8 \mathrm{CB}$-PFMC-vapor interface was extracted from XR measurements as a function of both the temperature $T-T_{\mathrm{NA}}$ of the $8 \mathrm{CB}$ subphase above the NA transition and the chemical potential $\Delta \mu \propto \Delta T_{\mu}=T-T_{\text {res }}$ of the PFMC film relative to the bulk liquid reservoir.

The 8CB side of the nematic-liquid (8CB-PFMC) interface is characterized by critical adsorption of interfaceinduced smectic layers. This is demonstrated by the observation of an electron density modulation whose period is equal to the known smectic layer spacing and whose amplitude decays exponentially toward the nematic subphase. The degree of induced smectic order, as measured by the amplitude decay length $\xi$, is independent of the amount of adsorbed PFMC. However, the measured $\xi$ increases as the NA transition temperature is approached from above, in quantitative agreement with the longitudinal correlation length $\xi_{\|} \propto(T$ $\left.-T_{\mathrm{NA}}\right)^{-0.7}$ for the smectic fluctuations in the bulk nematic. The results indicate that, just as in the case of the $8 \mathrm{CB}$-vapor interface, the $8 \mathrm{CB}$ molecules at the $8 \mathrm{CB}$-PFMC interface are oriented homeotropically such that the terminal methyl groups are at the interface with the fluorocarbon liquid.

The extracted electron density profiles further demonstrate that PFMC completely wets the $8 \mathrm{CB}$ surface. The measured thickness of the PFMC wetting film increases with decreasing $\Delta T_{\mu}$ according to the expected power law for 
complete wetting, $L \propto\left(\Delta T_{\mu}\right)^{-1 / 3}$. The wetting behavior of PFMC is nearly independent of the degree of interfacial smectic order in the subphase. The estimated values of the effective Hamaker constant $A_{\text {eff }}$, based on the observed $\Delta T_{\mu}$ dependence of $L$, are consistent with the dispersion interactions between hydro- and fluorocarbons.

\section{ACKNOWLEDGMENTS}

We thank Antonio Checco for helpful comments. The work at Brookhaven National Laboratory was supported by the U.S. Department of Energy, under Contract No. DEAC02-98CH10886. The work at Harvard was supported by the NSF Grant No. 03-03916.
[1] P. G. de Gennes, Rev. Mod. Phys. 57, 827 (1985).

[2] D. E. Sullivan and M. M. Telo da Gama, in Fluid Interfacial Phenomena, edited by C. A. Croxton (Wiley, New York, 1986), p. 45 .

[3] S. Dietrich, in Phase Transitions and Critical Phenomena, edited by C. Domb and J. L. Lebowitz (Academic, New York, 1988), Vol. 12, p. 1.

[4] B. M. Law, Prog. Surf. Sci. 66, 159 (2001).

[5] B. M. Ocko, X. Z. Wu, E. B. Sirota, S. K. Sinha, O. Gang, and M. Deutsch, Phys. Rev. E 55, 3164 (1997).

[6] J. Als-Nielsen, F. Christensen, and P. S. Pershan, Phys. Rev. Lett. 48, 1107 (1982).

[7] P. S. Pershan and J. Als-Nielsen, Phys. Rev. Lett. 52, 759 (1984).

[8] C. Rosenblatt, Phys. Rev. Lett. 53, 791 (1984).

[9] B. M. Ocko, A. Braslau, P. S. Pershan, J. Als-Nielsen, and M. Deutsch, Phys. Rev. Lett. 57, 94 (1986).

[10] P. S. Pershan, A. Braslau, A. H. Weiss, and J. Als-Nielsen, Phys. Rev. A 35, 4800 (1987).

[11] A. Braslau, Ph.D. thesis, Harvard University, Cambridge, 1988.

[12] B. M. Ocko, Phys. Rev. Lett. 64, 2160 (1990).

[13] Y. G. J. Lau, R. M. Richardson, and R. Cubitt, J. Chem. Phys. 124, 234910 (2006).

[14] J. Piris, M. G. Debije, N. Stutzmann, B. W. Laursen, W. Pisula, M. D. Watson, T. Bjornholm, K. Mullen, and J. M. Warman, Adv. Funct. Mater. 14, 1053 (2004).

[15] R. I. Gearba, D. V. Anokhin, A. I. Bondar, W. Bras, M. Jahr, M. Lehmann, and D. A. Ivanov, Adv. Mater. (Weinheim, Ger.) 19, 815 (2007).

[16] R. J. Bushby and O. R. Lozman, Curr. Opin. Solid State Mater. Sci. 6, 569 (2002).

[17] C. Bahr, Phys. Rev. E 73, 030702(R) (2006).

[18] P. Poulin, H. Stark, T. C. Lubensky, and D. A. Weitz, Science 275, 1770 (1997).

[19] J.-C. Loudet, P. Barois, and P. Poulin, Nature (London) 407, 611 (2000).

[20] I. I. Smalyukh, S. Chernyshuk, B. I. Lev, A. B. Nych, U. Ognysta, V. G. Nazarenko, and O. D. Lavrentovich, Phys. Rev. Lett. 93, 117801 (2004).

[21] F. P. Nicoletta, D. Cupelli, G. De Filpo, and G. Chidichimo, Appl. Phys. Lett. 84, 4260 (2004).

[22] J. M. Brake, M. K. Daschner, Y.-Y. Luk, and N. L. Abbott, Science 302, 2094 (2003).

[23] O. Gang, M. Fukuto, P. Huber, and P. S. Pershan, Colloids Surf., A 206, 293 (2002).

[24] M. Fukuto, O. Gang, K. J. Alvine, and P. S. Pershan, Phys. Rev. E 74, 031607 (2006).

[25] D. K. Schwartz, M. L. Schlossman, and P. S. Pershan, J. Chem.
Phys. 96, 2356 (1992).

[26] I. M. Tidswell, T. A. Rabedeau, P. S. Pershan, and S. D. Kosowsky, Phys. Rev. Lett. 66, 2108 (1991).

[27] R. K. Heilmann, M. Fukuto, and P. S. Pershan, Phys. Rev. B 63, 205405 (2001).

[28] M. Fukuto, Y. F. Yano, and P. S. Pershan, Phys. Rev. Lett. 94, 135702 (2005).

[29] O. Gang, K. J. Alvine, M. Fukuto, P. S. Pershan, C. T. Black, and B. M. Ocko, Phys. Rev. Lett. 95, 217801 (2005).

[30] F. J. Kahn, Appl. Phys. Lett. 22, 386 (1973).

[31] M. G. J. Gannon and T. E. Faber, Philos. Mag. A 37, 117 (1978).

[32] A. J. Leadbetter, J. C. Frost, J. P. Gaughan, G. W. Gray, and A. Mosley, J. Phys. (Paris) 40, 375 (1979).

[33] D. A. Dunmur and W. H. Miller, J. Phys. (Paris), Colloq. 40, C3-141 (1979).

[34] J. Thoen, H. Marynissen, and W. Van Dael, Phys. Rev. A 26, 2886 (1982).

[35] V. A. Korjenevsky and M. G. Tomilin, Liq. Cryst. 15, 643 (1993).

[36] P. DeSchrijver, W. VanDael, and J. Thoen, Liq. Cryst. 21, 745 (1996).

[37] D. Davidov, C. R. Safinya, M. Kaplan, S. S. Dana, R. Schaetzing, R. J. Birgeneau, and J. D. Litster, Phys. Rev. B 19, 1657 (1979).

[38] H. von Kanel and J. D. Litster, Phys. Rev. A 23, 3251 (1981).

[39] S. Sprunt, L. Solomon, and J. D. Litster, Phys. Rev. Lett. 53, 1923 (1984).

[40] J. D. Litster, J. Als-Nielsen, R. J. Birgeneau, S. S. Dana, D. Davidov, F. Garcia-Golding, M. Kaplan, C. R. Safinya, and R. Schaetzing, J. Phys. (Paris), Colloq. 40, C3-339 (1979).

[41] J. Als-Nielsen and D. McMorrow, Elements of Modern X-Ray Physics (Wiley, Chichester, U.K., 2001).

[42] S. K. Sinha, E. B. Sirota, S. Garoff, and H. B. Stanley, Phys. Rev. B 38, 2297 (1988).

[43] We also tried to fit by allowing $\phi_{L}$ to vary freely. However, for very thin wetting films $(L \lesssim 20 \AA)$, the parameters $\phi_{L}$ and $L$ were found to be strongly coupled and could not be independently determined with acceptably small uncertainties. On the other hand, for thick films $(L \gtrsim 20 \AA), \phi_{L}$ and $L$ are decoupled, and the best-fit value of $\phi_{L}$ agreed with the physically expected value of $\phi_{L}=\rho_{\mathrm{PFMC}} / \rho_{8 \mathrm{CB}}=1.59$ within its uncertainty. We therefore chose to use the fixed value of $\phi_{L}=1.59$ in the final fitting.

[44] B. M. Ocko, X. Z. Wu, E. B. Sirota, S. K. Sinha, and M. Deutsch, Phys. Rev. Lett. 72, 242 (1994).

[45] The upper-cutoff wave vector for the capillary fluctuations is expected to be limited by a molecular dimension $a$ parallel to the interface such that $q_{\max } \approx \pi / a[24,44]$. From the molecular 
weight and mass density of $8 \mathrm{CB}$ [33], the volume per $8 \mathrm{CB}$ molecule is $v_{0} \approx 490 \AA^{3} /$ molecule. Assuming that two $8 \mathrm{CB}$ molecules occupy a cylindrical volume of $2 v_{0}=(\pi / 4) a^{2} d$ where $d=31.7 \AA$ is the smectic layer spacing [32,37,40], one obtains an estimate of $a \approx 6 \AA$ or $q_{\max } \approx 0.5 \AA^{-1}$.

[46] The deviation $\xi<\xi_{\|}$at $T-T_{\mathrm{NA}}<0.1 \mathrm{~K}$ in Fig. 6(a) is likely due to the spread $\delta \alpha$ in the incident angle $\alpha$. Taking $\xi_{\max }$ $=1000 \AA$ as the saturation value for $\xi$, the half-width of the sharpest resolvable quasi-Bragg peak in $R / R_{F}$ would be given by $\delta q_{z} / 2 \approx(2 \pi / \lambda) \delta \alpha \approx \xi_{\max }^{-1}$, yielding $\delta \alpha \approx 0.24 \times 10^{-3} \mathrm{rad}$. In typical XR scans, the incident beam had an angular divergence of $\delta \alpha_{0}=0.14 \times 10^{-3} \mathrm{rad}$ and a vertical width of 0.05 $\mathrm{mm}$, corresponding to an illuminated footprint length of $f$ $=2 \mathrm{~mm}$ at $q_{z}=q_{0}=0.2 \AA^{-1}$. From this, we estimate the radius of curvature at the center of the $8 \mathrm{CB}$ surface to be of the order of $R \approx f /\left(\delta \alpha-\delta \alpha_{0}\right)=20 \mathrm{~m}$.

[47] E. B. Sirota, X. Z. Wu, B. M. Ocko, and M. Deutsch, Phys. Rev. Lett. 79, 531 (1997).

[48] M. Deutsch and B. M. Ocko (private communication).

[49] A fit to the vapor pressure curve of PFMC [J. S. Rowlinson and R. Thacker, Trans. Faraday Soc. 53, 1 (1957)] using the form $\ln (P)=-Q / R T+$ const yields $Q=33.4 \mathrm{~kJ} /$ mole for the latent heat of vaporization for PFMC. From the molecular weight and mass density of PFMC [R. B. Heady and J. W. Cahn, J. Chem. Phys. 58, 896 (1973)], the number density of liquid PFMC is estimated to be $n=5060$ and $4940 \mathrm{~mole} / \mathrm{m}^{3}$ at $T-T_{\mathrm{NA}}=0.7$ and $19.3 \mathrm{~K}$, respectively.

[50] Assigning the fitting parameter $L$ to the PFMC thickness (Sec. III C) implicitly assumes that the average plane of the PFMC$8 \mathrm{CB}$ interface is located at $z=0$, at which the error function part $\phi_{1}(z)+\phi_{3}(z)$ of the density profile [Eq. (2)] has a locally maximum slope. It is reasonable to question the validity of this assumption for very thin films for which the PFMC contribution to the profile becomes comparable to the overlap with the 8CB contribution [Figs. 4(d)-4(f)]. In view of this, we also extracted the PFMC thickness using an alternative approach that relies not on $z=0$ but on the relatively well-defined position $z_{1}=z_{0}+d / 4$ for the middle of the first $8 \mathrm{CB}$ smectic layer. The basic steps for this method can be summarized as follows. (i) The best-fit profile $\langle\rho(z)\rangle / \rho_{\infty}$ for the 8CB-PFMC-vapor interface [Figs. 4(d) -4 (f)] was integrated from $z=-\infty$ (vapor) to $z_{1}$, to yield $\Gamma_{8 / \mathrm{P} / \mathrm{v}}$, where the subscripts $8, \mathrm{P}$, and $\mathrm{v}$ represent $8 \mathrm{CB}$, PFMC and vapor, respectively. (ii) The $8 \mathrm{CB}$ contribution to $\Gamma_{8 / \mathrm{P} / \mathrm{v}}$ was estimated by performing the same integration on the profiles for the 8CB-vapor interfaces [Fig. 3(b)], which yields $\Gamma_{8 / \mathrm{v}}$. (iii) An effective PFMC thickness was obtained by defining $L_{\mathrm{eff}} \equiv\left(\Gamma_{8 / \mathrm{P} / \mathrm{v}}-\Gamma_{8 / \mathrm{v}}\right) / \phi_{L}$, where $\phi_{L}=\rho_{\mathrm{PFMC}} / \rho_{8 \mathrm{CB}}$ $=1.59$ (Sec. III A). At each $T$ and $\Delta T_{\mu}, L_{\text {eff }}$ thus obtained agrees with the fitting parameter $L$ within their scatter and uncertainties. However, the best-fit values for $L_{\mathrm{eff}}$ on the nematic (isotropic) subphase are slightly higher (lower) than those for $L$, resulting in a smaller discrepancy in the PFMC thickness between the two types of subphase than that for $L$ shown in Fig. 7(c). For example, $L_{\text {eff }}=21.4 \pm 0.4 \AA$ for the nematic subphase at $\left(T-T_{\mathrm{NA}}, \Delta T_{\mu}\right)=(0.7 \mathrm{~K}, 0.16 \mathrm{~K})$ and $L_{\text {eff }}$ $=23.6 \pm 2.3 \AA$ for the isotropic subphase at $(19.3 \mathrm{~K}, 0.18 \mathrm{~K})$ agree better than the corresponding values for $L$ (Table II). These details do not alter the main conclusions about the wetting behavior discussed in Sec. III C.

[51] J. Israelachvili, Intermolecular and Surface Forces (Academic, London, 1992). 